\title{
THE CALIBRATION AND SIMULATION OF THE GRB TRIGGER DETECTOR OF THE ULTRA FAST FLASH OBSERVATORY
}

\author{
M.-H.A. Huang ${ }^{1}$, S. Ahmad ${ }^{2}$, P. Barrillon ${ }^{2}$, S. Brandt ${ }^{3}$, \\ C. Budtz-Jørgensen ${ }^{3}$, A.J. Castro-Tirado ${ }^{4}$, S.-H. Chang ${ }^{5}$, Y.-Y. Chang ${ }^{6}$, \\ C.R. Chen ${ }^{5}$, P. Chen ${ }^{6}$, H.S. Choi ${ }^{7}$, Y.J. Choi ${ }^{8}$, P. Connell ${ }^{9}$, \\ S. Dagoret-Campagne ${ }^{2}$, C. Eyles ${ }^{9}$, B. Grossan ${ }^{10}$, J.J. Huang ${ }^{6}$, \\ S. Jeong ${ }^{11}$, A. Jung ${ }^{11}$, J.-E. Kim ${ }^{11}$, M.-B. Kim ${ }^{12}$, S.-W. Kim ${ }^{13}$, \\ Y.-W. Kim ${ }^{12}$, A.S. Krasnov ${ }^{14}$, J. Lee ${ }^{12}$, H. Lim ${ }^{12}$, C.-Y. Lin ${ }^{5}$, \\ E.V. Linder ${ }^{10}$, T.-C. $\mathrm{Liu}^{6}$, N. Lund ${ }^{3}$, K.W. Min ${ }^{8}$, G.-W. Na ${ }^{11}$, \\ J.-W. Nam ${ }^{6}$, M.I. Panasyuk ${ }^{14}$, I.H. Park ${ }^{12}$, V. Reglero ${ }^{9}$, J. Ř́pa ${ }^{12}$, \\ J.M. Rodrigo ${ }^{9}$, G.F. Smoot ${ }^{10}$, J.-E. Suh ${ }^{11}$, S. Svertilov ${ }^{14}$, N. Vedenkin ${ }^{14}$, \\ M.-Z. Wang ${ }^{6}$ and I. Yashin ${ }^{14}$
}

\footnotetext{
${ }^{1}$ Department of Energy Engineering, National United University, Miao-Li, Taiwan; e-mail: mahuang@nuu.edu.tw

${ }^{2}$ Laboratoire de l'Accélérateur Linéaire, University of Paris-Sud 11, Orsay, France

3 Technical University of Denmark, Copenhagen, Denmark

${ }^{4}$ Instituto de Astrofisica de Andalucía, CSIC, Granada, Spain

${ }^{5}$ National Space Organization, Taiwan

${ }^{6}$ LeCosPA, National Taiwan University, Taipei, Taiwan

7 Korea Institute of Industrial Technology, Cheonan, Korea

8 Department of Physics, Korea Advanced Institute of Science and Technology, Daejeon, Korea

9 University of Valencia, Valencia, Spain

10 Institute for the Early Universe, Ewha Womans University, Seoul, Korea

11 Department of Physics, Ewha Womans University, Seoul, Korea

12 Sungkyunkwan University, Suwon, South Korea

13 Department of Astronomy, Yonsei University, Seoul, Korea

14 SINP, Moscow State University, Moscow, Russia
} 


\begin{abstract}
The UFFO (Ultra-Fast Flash Observatory) is a GRB detector on board the Lomonosov satellite, to be launched in 2013. The GRB trigger is provided by an X-ray detector, called UBAT (UFFO Burst Alarm \& Trigger Telescope), which detects X-rays from the GRB and then triggers to determine the direction of the GRB and then alerts the Slewing Mirror Telescope (SMT) to turn in the direction of the GRB and record the optical photon fluxes. This report details the calibration of the two components: the MAPMTs and the YSO crystals and simulations of the UBAT. The results shows that this design can observe a GRB within a field of view of $\pm 35^{\circ}$ and can trigger in a time scale as short as $0.2-1.0 \mathrm{~s}$ after the appearance of a GRB X-ray spike.
\end{abstract}

\title{
1 Introduction
}

The UFFO (Ultra-Fast Flash Observatory) is a GRB detector, designed to catch the optical photons as early as possible. The UFFO-path finder is the first detector, which consists of two sub-detectors: the Slewing Mirror Telescope (SMT) and the UFFO Burst Alert Telescope (UBAT) (Park et al. 2013). UBAT uses a coded mask detector to detect X-rays from a GRB (Kim et al. 2012). The triggered pattern is used to determine the direction from which the GRB arrives and then the location of the GRB is sent to the SMT. SMT consists of a slewing mirror in front of an optical telescope. By turning this mirror, the field of view can be extended from the $17^{\prime} \times 17^{\prime}$ of a telescope and CCD to almost $35^{\circ} \times 35^{\circ}$ (Jeong et al. 2013). It takes only approximately $1 \mathrm{~s}$ to move the mirror to the desired direction. The UFFO-path finder is installed on the Lomonosov satellite and is scheduled to be launched in 2013 .

This study firstly constructs a complete model of UBAT in a GEANT4 simulation package and then studies several key characteristics of the UBAT. Section 2 describes UBAT geometry and the GEANT4 model. Section 3 presents the results from the calibration of the MAPMTs and the YSO crystals. Section 4 presents simulation results, including the active energy range for X-rays, the potential noises from charged particles and the determination of the direction of the GRB and the angular resolution.

\section{UBAT structure and model}

The top of UBAT is a $68 \times 68$ pixels coded mask, made from $1 \mathrm{~mm}$ thick Tungsten. In order to increase the mechanical strength, Kapton tapes are glued to the both sides of the coded mask. At the base of UBAT is an $6 \times 6$ array of $\mathrm{X}$-ray detectors, which consists of YSO crystals coupled with Multi-Anode Photo-Multiplier Tubes (MAPMT) and readout electronics. The YSO crystals convert incoming X-rays into optical photons and then the MAPMTs convert the photons to photoelectrons and amplify the current to render it detectable. In order to isolate the optical 
photons within one pixel, each YSO crystal is wrapped with a thin reflecting tape, except for the side facing the MAPMT.

\section{MAPMT and YSO crystal calibration}

The 64 channels MAPMT are custom made by Hamamatsu Photonics, with $8 \times$ 8 pixels, $2.88 \mathrm{~mm}$ square, beneath a $0.8 \mathrm{~mm}$ thick frontal UV glass. The gain of each pixel of the MAPMT is defined as the DAQs (Data AcQuisition system) reconstructed voltage reading per photoelectron (PE) emitted from the photocathode. The NuTel DAQ system is used (Yeh et al. 2004), which is capable of simultaneously taking data from 512 channels, to calibrate the gain of the MAPMTs. A blue LED is used, which has the same wavelength as that has emitted by the YSO. This sends a pulsed light to test the MAPMT's responses at different high voltages. The DAQ records the data by synchronizing with the LED pulse, instead of the MAPMT signals. The signals $S(x)$ as a function of DAQ reading $x$ in $\mathrm{mV}$ are then fitted by a Poisson functions, $S(x)=A \times \mu^{x / G} \times e^{-\mu} / \Gamma((x / G)+1)$, to determine the gain $G$ and mean number of photo-electrons $\mu$. Figure 1 shows a typical light pulse histogram with its fitting result for one channel and the gain for all channels.
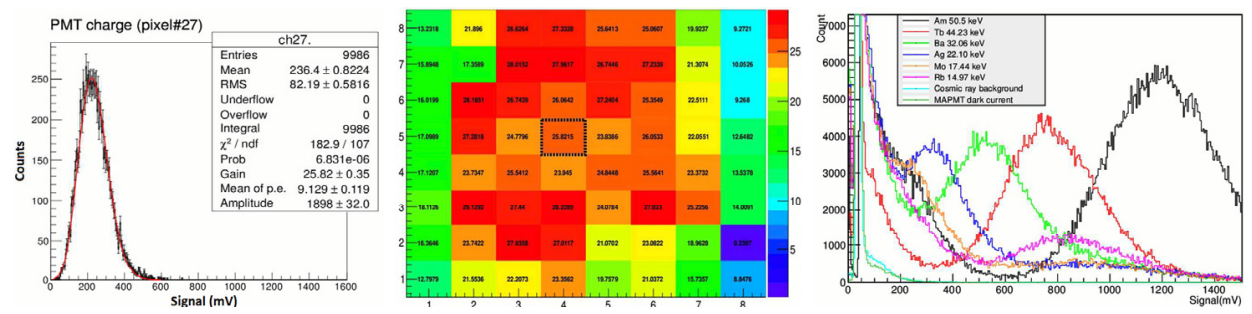

Fig. 1. The figure on the left side, shows a histogram of the data from channel 27 . The figure on the center shows the Gain distributions of this MAPMT at 800V. Channel 27 is located at coordinate index $(4,5)$ and surrounded by a black dash square. The figure on the right side shows the energy calibration for the YSO crystals.

The scintillator is an array of 64 small YSO crystal blocks, each of size $2.65 \mathrm{~mm} \times$ $2.65 \mathrm{~mm} \times 3 \mathrm{~mm}$ (thickness), which has the same dimensions as the MAPMT and which attaches to its frontal UV glass. For the calibration of the YSO crystals, a variable X-ray source is placed in front of the YSO and MAPMT assembly and the signals from each cell are recorded. Figure 1 shows a combination of several X-ray emission lines. The YSO photon yield is derived as 10.5 photons $/ \mathrm{keV}$ and the light yield is linear in the test range, from $22.1 \mathrm{keV}$ to $59.5 \mathrm{keV}$.

\section{UBAT simulation}

In order to simulate the operation of UBAT, a complete model of UBAT was constructed, using the GEANT4 simulation package. The coded mask, shown in 
panel (a) of Figure 2, has an approximately 50\% opening fraction and a crossing pattern of reinforcement rigs. The Kapton tape cuts off X-rays below approximately $5 \mathrm{keV}$ and the Tungsten cuts off X-rays below approximately $200 \mathrm{keV}$. Between $5 \mathrm{keV}$ and $200 \mathrm{keV}$, X-ray photons trigger UBAT and produce a certain pattern on $48 \times 48$ detector pixel array, which then can be used to determine the direction of arrival of the X-rays, using a reconstruction algorithm (Connel et al. 2013). Figure 2 shows the patterns for three directions, relative to the main axis of the UBAT.

(a) Coded Mask Pattern

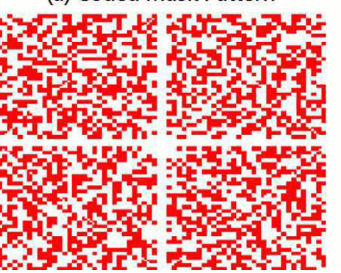

(b) Angle $(\theta=0, \phi=0)$

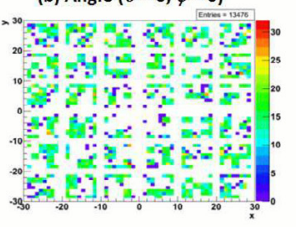

(c) Angle $(O=30, \phi=0)$

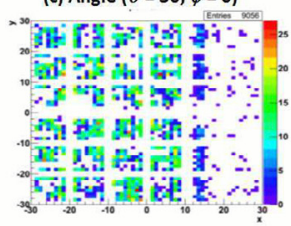

(d) Angle $(\theta=20, \phi=45)$

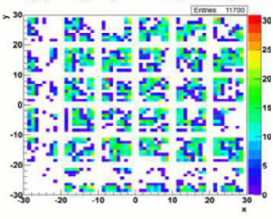

Fig. 2. Panel (a) shows UBAT coded mask pattern. Red represents open cells and white represents the blocked cells and the supporting rigs. The other three panels (b, c, d) show the patterns of triggered cells, from three directions. The color coding denotes the charge of the triggered pixels.

Several GRB light curves are used to study the trigger time. The mean X-ray count before the GRB is considered to be the background noise and counts above background are treated as a GRB signal. Simulations were performed at one frame per second and both photons from the background and/or GRB were generated independently. Each $1 \mathrm{~s}$ frame can be stack together to form $2 \mathrm{~s}, 4 \mathrm{~s}, 8 \mathrm{~s}, 16 \mathrm{~s}, 32 \mathrm{~s}$ image frame continuously. The trigger rate of those frames of different period are recorded and then the running mean and RMS values are calculated (Kim et al. 2012). If a frame has a rate greater than a predefined level, then it passes the first level trigger, called the rate trigger, and the pattern is then analyzed to determine the direction of the arrival. If the arrival direction can be determined above some predefined SNR, it then passes the second level trigger, called the image trigger. This simulation accounts for all the blockage from the supporting structure and for the stochastic nature of the background noise and the signal.

Apart from the astronomical X-ray background, the charged particles from cosmic rays and space radiation also contribute to noise. These effects are studied by injecting protons, electrons and gamma rays of various energy ranges, from $10 \mathrm{keV}$ to $1 \mathrm{TeV}$. Low-energy charged particles cannot penetrate the $1 \mathrm{~mm}$ Tungsten coded mask, they could penetrate through the opening holes and trigger some pixels. However, electron flux are almost constant within minutes and will not pass the rate trigger, even a large random fluctuation may cause a rate trigger, their isotropic distribution may not pass the image trigger. Although high-energy particles can penetrate the sidewalls, their signals are concentrated in just one frame and their trails are mainly grouped in near-by pixels, which is inconsistent 
with the pattern of the coded mask. Therefore, it is concluded that charged particles are not a major source of noise or can be excluded by identifying the particular abnormal detector pixel pattern they leave behind.

Approximately 300 GRB light curves from BATSE were used in the trigger simulation. For a strong GRB, UBAT can trigger $0.5 \mathrm{~s}$ after the GRB starts and an additional second is required to slew the SMT. The UFFO can record optical photons as early as $1.5 \mathrm{~s}$. The exact time depends on the signal-to-noise ratio.

Once a rate trigger occurs, the image pattern is analyzed to determine the arrival direction of the X-ray source. The angular resolution is determined by comparing the input direction of the X-rays to the reconstructed direction. Figure 3 shows the angular error distribution in three energy ranges, assuming an isotropic distribution of X-ray sources. The mean angular errors are within $17^{\prime}$ or $0.283^{\circ}$ of the field of view of the SMT telescope and only a few percent of events are outside this limit, at a higher energy. The higher the photon energy, the larger is the mean angular error. However, in a realistic GRB spectrum, there are much less high-energy photons than low-energy photons. It is concluded that UBAT can localize GRB sources within the design values.
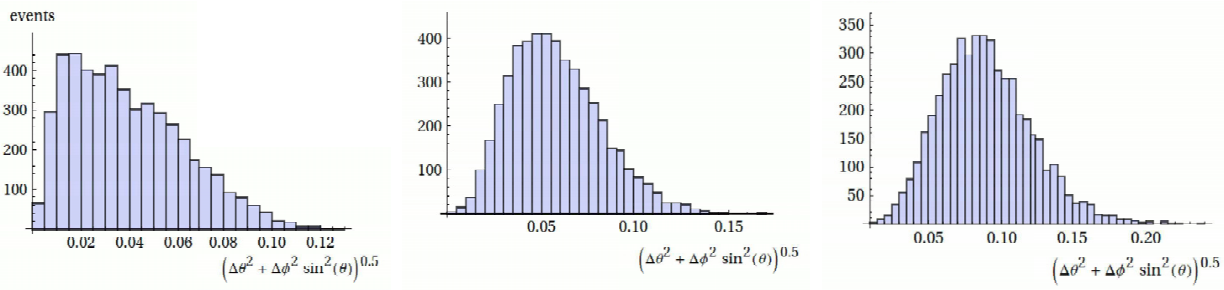

Fig. 3. The distributions of the angular error for 5, 50, and $100 \mathrm{keV}$ photons are shown, from left to right. The horizontal axis represents the angular error, in degree. The input directions are isotropically distributed.

\section{References}

Connell, P., Ahmad, S., Barrillon, P., et al., 2013, for the UFFO collaboration, proc. 2012, Gamma Ray Burst Symposium (Marbella, Spain) (Oct. 8-12, 2012)

Jeong, S., Nam, J.W., Ahn, K.B., et al., 2013, for the UFFO collaboration, Opt. Express, 21,2263

Kim, J.E., Ahmad, S., Barrillon, P., et al., 2012, for the UFFO collaboration, Proc. SPIE 8443, 84432R; doi:10.1117/12.926348

Park, I.H., Ahmad, S., Barrillon, P., et al., 2013, for UFFO collaboration, submitted to the New J. Phys.

Yeh, P., Athar, A., La Barbera, N., et al., 2004, Mod. Phys. Lett. A., 19, 1117 